\title{
Function, energy and environment orientation in innovation research of rare earth materials
}

\author{
ZHOU XueZhen, LI HongYang, LIU YanZhu \& LI YongXiu ${ }^{*}$ \\ Research Center for Rare Earths \& Micro/nano Functional Materials, Nanchang University, Nanchang 330031, China
}

Received November 11, 2019; accepted November 25, 2019; published online November 28, 2019

Citation: Zhou X Z, Li H Y, Liu Y Z, et al. Function, energy and environment orientation in innovation research of rare earth materials. Sci China Tech Sci,
2019, 62: 2302-2305, https://doi.org/10.1007/s11431-019-1490-y

Rare earths (REs), as a kind of key elements widely used in high-tech industry, have been paid more and more attention all over the world. At a time when the world trade war is raging, dignitaries and executives are trying to control this strategic resource, while scientific and technological researchers are trying to solve the scientific and technological problems in the development of RE industry. The innovation research of RE materials are orientated by three main aspects as shown in Figure 1: one is to make the production process of RE materials more green and sustainable, the second is to strengthen the material properties and save energy, and the third is to find new functional materials with improved quality.

The leading technology of RE extraction and separation is mainly mastered by China, but it does not meet the requirements of green chemistry and processing. There are some unsatisfactory problems in the separation and metallurgy of RE resources [1,2], and there is still room for compression of the consumption of separation process and the discharge of pollutants.

The reported recovery rate of ore dressing is between $50 \%$ and $70 \%$ for rock mineral RE resources. In the light of the problems of low recovery rate and comprehensive utilization of the RE resources in Baotou, new processes of separating RE minerals from the tailings and comprehensively recovering the associated resources are carried out [1].

The reported yield of ion adsorption rare earth is between

\footnotetext{
*Corresponding author (email: yxli@ncu.edu.cn)
}

$72 \%$ and $80 \%$. The key problems resulting serious environment pollution were determined to be the blind promotion policy of in-situ leaching technology which is difficult to control the seepage during leaching process [2]. The use of excessive ammonium sulfate has led to a large number of pollutants and the occurring of landslides. Therefore, aluminium sulfate, which is produced from aluminum containing slag, is put forward as a high efficiency and green leaching reagent [2-4]. Combined with the controlled heap leaching process and environmental engineering mode, a new generation of technology with high-efficiency, no-pollution, no RE loss has been proposed.

The progress of RE separation technology is mainly manifested in the successful application of technologies which have made the product quality improved, the consumption saved and the pollutant greatly reduced. Such as P507-isooctanol separation system for heavy RE [5], hyperlink extraction [6], organic phase RE continuous saponification, RE precipitation and full recycling of waste water. In addition, the proposal of a new system for extraction separation of associated uranium and thorium has laid a foundation for addressing the issue of radioactive contamination and developing nuclear energy materials in the future [7].

Magnetic material is the largest application field of RE [8], particularly in energy and energy saving fields. In recent years, with the increasing application of sintered $\mathrm{Nd}-\mathrm{Fe}-\mathrm{B}$ in high performance motor, high magnetic energy product and high working temperature magnet has become the core goal 


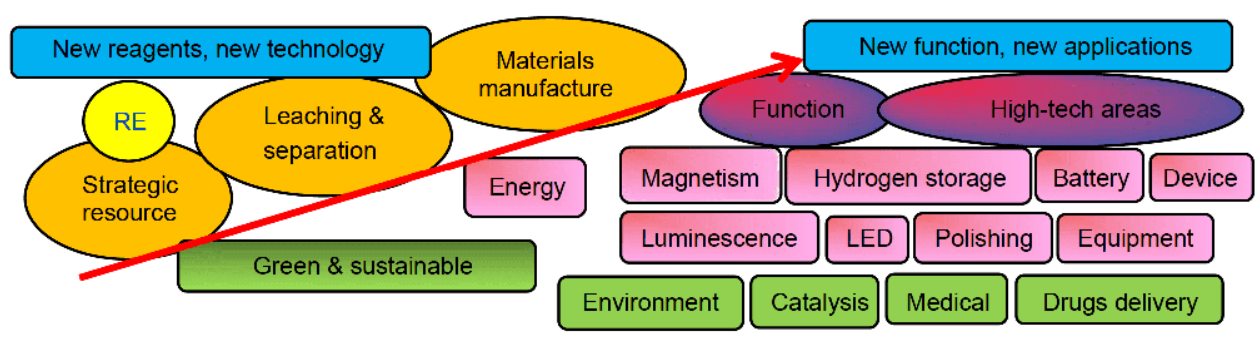

Figure 1 (Color online) The orientation in innovation research of rare earth materials.

of research and development ( R \& D) and the results are remarkable. In order to balance the utilization of RE resources and reduce the cost, magnets containing high abundance RE have become an important goal of R \& D. The new techniques include grain boundary diffusion, grain boundary regulation and double (multiple) alloys (including double main phase), as well as grain refinement methods aimed at high coercive force of near single domain particles.

Hydrogen storage alloys are very important in hydrogen energy vehicles and $\mathrm{Ni}-\mathrm{H}$ batteries. Recent researches have focused on the gaseous sorption and electrochemical hydrogen storage properties of RE alloys in order to improve their storage capacity, kinetics, thermodynamics, electrochemical performance [9]. Although the application of RE hydrogen storage materials in batteries is challenged and squeezed by lithium ion batteries, its safety performance in large capacity power batteries is better, and has great potential in cold regions and military equipment. Therefore, the $\mathrm{R} \& \mathrm{D}$ of new alloy types and their applications will have better development. For example, yttrium magnesium alloy not only has good hydrogen storage performance and battery application potential, but also has great application potential in transportation equipment lightweight and medical materials.

The once brilliant RE lamp phosphor is weakening due to the rise of LED lighting. The global annual demand is only $1200 \mathrm{t}$, including $400 \mathrm{t}$ fluorescent powder for LED lighting. In full spectral lighting, high stability red and cyan phosphors are needed, the stability of current nitride and fluoride red powder still needs to be improved. The research of RE luminous materials mainly turns to two main aspects: one is the high quality growth and manufacture of large bulk materials, including optical functional crystal material (OFCM) and transparent ceramics. Under the guidance of the new theory, the application of new manufacturing technology has greatly improved the performance price ratio [10]. OFCM is a kind of structure-function integrated material which realizes high transmittance and optical function of single crystal or polycrystalline ceramic system by microstructure regulation and RE ion doping. Its appearance not only enriches the research connotation of material science, but also expands the application of OFCM in information, energy, medical treatment, advanced manufacturing and national security. As typical representatives of OFCM, laser materials and flicker materials play an irreplaceable role in high-end equipment manufacturing, high-energy laser weapons, laser fusion ignition, nuclear medical imaging, high-energy physical detection and national security detection $[10,11]$.

The second is the synthesis of nano-phosphors and their applications. Over the past years, there has been a vast growth in the RE luminescence inorganic materials with cation or anion substitution, including molybdates, tungstates, vanadates, phosphates and oxides. The solid-state synthesis is most commonly applied to obtain RE phosphors. However, the hydrothermal synthesis is frequently used to construct $0 \mathrm{D}$ to $3 \mathrm{D}$ hierarchical structures. By doping different activator ions, and different combinations and concentrations of lanthanides, materials yielding a wide range of emission colors (red, green, blue, yellow and white) could easily be obtained [11]. For example, $\mathrm{RE}^{3+}$-doped fluoride luminescent materials that bear advantageous properties for use in photonic and biophotonic applications like white light emitting materials, photodynamic therapy, FRET-based and other biological detections, in vivo and in vitro imaging. The luminescence property can be effectively tuned by varying the crystal phase, shape, size and lattice strain of the host materials and by surface modification/functionalization. However, despite a substantial progress in $\mathrm{RE}^{3+}$-doped fluoride materials, it is still a long way to go for successful applications [12]. Recently, $\mathrm{Nd}^{3+}$-sensitized upconversion nano particles (UCNP) which can be excited with $808 \mathrm{~nm}$ has been widely investigated as alternative nanoparticles that can surmount the overheating effect which exists in $\mathrm{Yb}^{3+}$ sensitized UCNP due to its $980 \mathrm{~nm}$ excitation wavelength overlaps with the absorption band of water molecules in the biological environment [13]. Meanwhile, luminescent nanoparticles are applied for the plant imaging in vivo and in vitro, while the plant photosynthesis is dependent on the specific light wavelength, providing the luminescent nanoparticles an opportunity to optimize the agriculture light $[13,14]$.

There are many reports on synthesis, structure, performance characterization and device application of RE complex fluorescent materials. The application of optical 
conversion agricultural film has been widely promoted. In electroluminescent devices or kneading collapsible OLED devices, the synthesis and assembly of RE complexes with high stability and high luminous efficiency are still challenges [15].

The highly stable coordination compounds and nanoparticles of $\mathrm{Gd}(\mathrm{Eu}, \mathrm{Tb}, \mathrm{Dy})$ are the main research objects of magnetic resonance imaging (MRI) reagents [16]. Magnetic, photography and their medical functions can be combined to develop a multi-model detection system and drug delivery process. Efforts to develop MRI-detectable biomolecules have focused on proteins transporting paramagnetic metals for $T_{1}$ and $T_{2}$ relaxation enhancement or containing large numbers of exchangeable protons for chemical exchange saturation transfer. While these pioneering developments established several key uses of biomolecular MRI, such as imaging of gene expression and functional biosensing [17].

Lanthanide complexes mediated coordinative chain transfer polymerization of conjugated dienes features catalyst economy, well-controlling over both microstructure and architecture of the resulting polymers, and accessibility for novel (co)polymers [18]. Tremendous advances have been witnessed in the past few years and represent a novel strategy to prepare polydiene synthetic rubbers with controlled high molecular weight and narrow molecular weight distribution, which has reached the practical industrial application level, demonstrating a great potential in the future.

The catalytic application of RE has been a concern, especially in the catalytic conversion process related to pollutants $\left(\mathrm{NO}_{x}, \mathrm{SO}_{x}\right)$. Magnesium aluminate spinel containing cerium is the most effective catalyst for removing $\mathrm{SO}_{2}$ from catalytic cracking flue gas. This series of catalysts has strong sulfur resistance and toxicity in $\mathrm{SO}_{2}$ and has obvious activity in the reduction of $\mathrm{NO}_{x}$ by $\mathrm{CO}$. It can effectively control the emissions of $\mathrm{SO}_{2}$ and $\mathrm{NO}_{x}$ in flue gas at the same time, but it is necessary to deal with $\mathrm{H}_{2} \mathrm{~S}$ released from the reaction.

The catalysts involved in the catalytic reduction of $\mathrm{SO}_{2}$ in flue gas are perovskite RE complex oxides, fluorite RE complex (mixed) oxides. The advantages of $\mathrm{CO}$ reduction method are that elemental sulfur can be recovered, the source of reductant $\mathrm{CO}$ is convenient, and usually exists in flue gas, which can fundamentally control the pollution caused by $\mathrm{SO}_{2}$ and has the prospect of industrialization. With the environmental protection requirements increasingly stringent, the development of advanced RE catalytic materials and automobile exhaust purification technology is still a hot spot in the field of RE [19].

The application of polishing materials increases rapidly with the development of preparation and application technologies. For example, application in high precision processing of liquid crystal display, mobile phone screen and other displays makes its market expand rapidly. The im- provement of polishing powder production technology is mainly reflected in the control technology of production process, especially the production of high quality RE carbonate precursor makes the polishing materials more fine, the process is shorter, the energy consumption is lower, and the pollutant production is smaller. For example, the spherical and surface electrical adjustment technologies of polishing powder based on the doping and surface modification technology which takes RE carbonate and basic RE carbonate as precursors.

With the continuous reduction of chip feature size and the improvement of chip integration, chemical mechanical polishing (CMP) technology is put forward higher requirements. The preparation of CMP semiconductor wafer polishing slurry from nano-cerium oxide with controllable particle size has become an important component of semiconductor wafer polishing research and application.

The utilization of RE functional properties is the main way to develop new RE materials [20]. Although it cannot be expected that a new material with high application value and wide range can be introduced in a short term, this hope still exists. Therefore, there are many researches on the synthesis and application properties of RE compounds with controlable physical characteristics, there are many publicly reported results in chemistry, physics, materials, environment, energy, electronics, information, aerospace and so on. There may be many unreported results, because they have more practical value and potential, so it is incomplete to rely solely on publicly reported results to comment on current progress.

This work was supported by the National Natural Science Foundation of China (Grant No. 51864033), the Key $R \&$ D Program of the Ministry of Science and Technology (Grant No. 2019YFC0605000), and Major Projects of Consulting and Research of the Chinese Academy of Engineering (Grant No. 2018-ZD-11).

1 Yang Z F, Ma Y, Wang Y. Rare Earth Mining and Processing and Environmental Protection. Beijing: Metallurgical Industry Press, 2018

2 Li Y X. Ion Adsorption Rare Earth Resources and Their Green Extraction. Beijing: Chemical Industrial Press, 2014

3 Yang L, Wang D, Li C, et al. Searching for a high efficiency and environmental benign reagent to leach ion-adsorption rare earths based on the zeta potential of clay particles. Green Chem, 2018, 20: 45284536

$4 \mathrm{Xu}$ Q, Sun Y, Yang L, et al. Leaching mechanism of ion-adsorption rare earth by mono valence cation electrolytes and the corresponding environmental impact. J Clean Prod, 2019, 211: 566-573

5 Li D. Development course of separating rare earths with acid phosphorus extractants: A critical review. J Rare Earths, 2019, 37: 468-486

6 Cheng F X, Wu S, Liao C S, et al, Review and recent progresses on theory of countercurrent extraction. J Chin Soc Rare Earths, 2017, 35 : $1-8$

7 Kuang S T, Liao W P. Progress in the extraction and separation of rare earths and related metals with novel extractants: A review. Sci China Tech Sci, 2018, 61: 1319-1328

$8 \mathrm{Hu}$ B P, Rao X L, Niu E, et al, Technological progress and industrial development of rare earth permanent magnet materials. Chin Mater Prog, 2018, 37: 653-661 
9 Liang F, Lin J, Cheng Y, et al. Gaseous sorption and electrochemical properties of rare-earth hydrogen storage alloys and their representative applications: A review of recent progress. Sci China Tech Sci, 2018, 61: 1309-1318

10 Sun C T, Xue D F. Pulling growth technique towards rare earth single crystals. Sci China Tech Sci, 2018, 61: 1295-1300

11 Liu J, Kaczmarek A M, Van Deun R. Advances in tailoring luminescent rare-earth mixed inorganic materials. Chem Soc Rev, 2018, 47: 7225-7238

12 Sharma R K, Mudring A V, Ghosh P. Recent trends in binary and ternary rare-earth fluoride nanophosphors: How structural and physical properties influence optical behavior. J Lumin, 2017, 189: 44-63

13 Dibaba S T, Xiaoqian Ge S T, Ren W, et al. Recent progress of energy transfer and luminescence intensity boosting mechanism in $\mathrm{Nd}^{3+}$. sensitized upconversion nanoparticles. J Rare Earths, 2019, 37: 791805

14 Dong R, Li Y, Li W, et al. Recent developments in luminescent nanoparticles for plant imaging and photosynthesis. J Rare Earths, 2019,
37: 903-915

15 Wei C, Ma L, Wei H B, et al. Advances in luminescent lanthanide complexes and applications. Sci China Tech Sci, 2018, 61: 12651285

16 Zhang L, Li J J, Liu K. Recent advances in gadolinium-based MRI metal responsive agent. Sci China Tech Sci, 2018, 61: 1329-1333

17 Mukherjee A, Davis H C, Ramesh P, et al. Biomolecular MRI reporters: Evolution of new mechanisms. Prog Nucl Magn Reson Spectr, 2017, 102-103: 32-42

18 Wang F, Liu H, Hu Y M, et al. Lanthanide complexes mediated coordinative chain transfer polymerization of conjugated dienes. Sci China Tech Sci, 2018, 61: 1286-1294

19 Weng D, Ran R, Cao Y D, et al. Research progress on ceria-based rare earth catalytic materials used for air pollution control. Mater China, 2018, 37: 756-764

20 Li W, Huang X, Huang W. Effects of $\mathrm{Ca}, \mathrm{Ag}$ addition on the microstructure and age-hardening behavior of a $\mathrm{Mg}-7 \mathrm{Sn}$ (wt\%) alloy. Mater Sci Eng-A, 2017, 692: 75-80 